\title{
Do Maternal Demographics and Prenatal History Impact the Efficacy of Betamethasone Therapy for Threatened Preterm Labor?
}

\author{
Mary Kinney ${ }^{1}$, David M. Haas ${ }^{2}$, Hayley Trussell ${ }^{1}$, Larissa Silva', Sara Quinney ${ }^{2}$ \\ ${ }^{1}$ Indiana University School of Medicine; ${ }^{2}$ Indiana University School of Medicine, Department of \\ Obstetrics and Gynecology
}

Background/Objective: Betamethasone is used to accelerate fetal lung maturation in women with threatened preterm labor, but its efficacy is variable and limited by the lack of patient individualization in its dosing and administration. To determine sources of variability and potential opportunities for individualization of therapy, the objective of this study was to evaluate maternal factors associated with development of neonatal respiratory distress syndrome (RDS) in a cohort of women who received betamethasone.

Methods: This study prospectively enrolled women, gestational ages 23-34 weeks, who received betamethasone for threatened preterm labor $(n=208)$. Maternal demographics, prenatal history, and neonatal outcomes were abstracted from Epic and Cerner records. RDS was the primary outcome. Associations between RDS diagnosis and factors such as maternal demographics, prenatal history, and betamethasone dosing were evaluated in a multivariable regression adjusted for gestational age at delivery. A secondary analysis limited the cohort to women who delivered within 2 weeks of betamethasone dosing ( $n=95)$.

Results: Of 208 deliveries, $44.1 \%$ resulted in neonatal RDS. Within the overall cohort, the only significant association with RDS was the type of delivery, with $61.3 \%$ of cesarean births resulting in RDS versus $28.7 \%$ of vaginal births (adjusted OR 1.17 [1.06-1.28]). Among deliveries within 14 days of betamethasone dosing, women who experienced preterm premature rupture of membranes (PPROM) had lower RDS outcome rates than those without PPROM (52.6\% vs. $78.9 \%$, adjusted OR 0.80 [0.65-0.98]). Maternal age, BMl, race, and ethnicity were not associated with RDS.

Conclusion: Maternal characteristics alone may not be useful biomarkers in predicting neonatal RDS. The association between PPROM and RDS may suggest the importance of the time frame between betamethasone dosing and delivery. The finding of higher risk for RDS among neonates born by cesarean is consistent with other studies and bears further exploration as betamethasone therapy may mediate the association. 